patient management, including monitoring, escalation and medical reviews, in addition to staffing issues, debriefs and parental feedback. Areas for improvement were shared across services by monthly newsletters and updates at teaching sessions and departmental risk meetings.

Results Between November 2016 and September 2017, 26 children aged 2 days to 15 years were transferred to PICU, of which 69\% were from the Emergency Department. The primary problem was respiratory failure in $50 \%$ of cases.

In the first 6 months many cases had incomplete Paediatric Early Warning Score (PEWS) monitoring and/or poor escalation of care documentation. Shared learning and teaching on PEWS resulted in recent cases of exemplary practice. Initially EDRM was incomplete for patients moved to theatre from the emergency department for intubation. Recommendation to scan notes prior to moving resulted in complete EDRM records in all subsequent reviews. The multidisciplinary team recognised that parental views remained unheard and so initiated parents being offered the Friends and Family feedback card post-PICU repatriation.

Conclusion Shared learning from regular structured multidisciplinary case note reviews of PICU transfers results in improvements in the management, safety and documentation of children presenting with critical illness.

\section{G395(P) AN INNOVATIVE APP DESIGNED TO REDUCE HEALTHCARE-RELATED ANXIETY IN YOUNG CHILDREN}

${ }^{1} \mathrm{R}$ Duncombe, ${ }^{2} \mathrm{R}$ Evans Fry. 'Paediatrics, Oxford University Hospitals NHS Trust, Oxford, UK; ${ }^{2}$ Paediatrics, Bucks Healthcare NHS Trust, Aylesbury, UK

\subsection{6/archdischild-2018-rcpch.384}

Aims To create an interactive App that reduces healthcare associated anxiety in young children through education and play.

Method A significant number of children have contact with healthcare in the UK every year, with 3 million children attending $\mathrm{A}$ and $\mathrm{E}$, and 1 in 10-15 children being admitted to hospital. The literature suggests that $20 \%$ of children admitted to hospital suffer some form of emotional or behavioural disturbance. Play is key to childhood development and learning, and is practiced across healthcare settings. Psychological preparation for healthcare experiences has been shown to help reduce patient and parental anxiety. With ever-increasing budget restrictions within the NHS, innovative ideas are required to tackle this problem alongside invaluable play therapy resources.

We created an interactive App for children aged 4-8 years designed to ameliorate distress and improve paediatric healthcare experiences. It familiarises children with hospital environments and the common scenarios they may encounter through interactive stories, games and animations.

A patient and carer survey was undertaken following Phase 1 development of the app (a single character story with animations and games). Twenty children aged 4-8 years were selected at random to play on the App (for up to $20 \mathrm{~min}$ ) with carer supervision. Following this standardised questionnaires were completed by the child (visual rating scales) and carer to assess educational value and usability.
Results The overall feedback from study participants was good with $100 \%$ reporting that they would download the final product and 95\% believing that the completed App would be useful for educating children about hospitals.

Since the release of the App there has been 350 downloads within the first two weeks and positive feedback received via App ratings, social media messages and emails.

Conclusions New and innovative ideas are imperative to develop NHS services for the future. This is an illustration of how digital technology using play, can help to improve Paediatric healthcare experiences and potentially increase the efficiency of a service. A larger study of the beneficial effects of this App is planned following its wider distribution within the NHS.

\section{G396(P) NEONATAL 'VIRTUAL' CLINIC: A QUALITIY IMPROVEMENT PROJECT}

L Fullerton, C Cane. Neonatal Department, Royal Free NHS Foundation Trust, Barnet Hospital, London, UK

\subsection{6/archdischild-2018-rcpch.385}

Aims To improve the follow up of outstanding jobs from the neonatal unit and post-natal ward. Outstanding jobs were often being left for prolonged periods with no dedicated person to follow up and with no record of jobs having been completed. This resulted in poor patient care, inadequate communiation and potential for clinical risk.

Methods A quality improvement project was initiated and a plan, study, do, act cycle was used. Initial review showed that oustanding jobs were put onto the bottom of the clinical handover list and random junior doctors were irreguarly asked to review it during their clinical time. Some jobs had been present for more than a 3 month period with most jobs having inadequate information detailing reasons required for follow up. A number of jobs were inappropriate for junior doctor follow up thereby identifying a lack of education and under utilisation of pathways already in place. We set up a weekly neonatal 'virtual' clinic and amended the rota in conjunction with management and previously allocated admin time for the registrars. Aims and rules of the list were created and the junior doctors were educated as to the changes. A registrar was assigned during their admin time to run a 'virtual' phone clinic, once a week, where they would chase results and contact parents and/or other health professioanls whilst documenting their actions on electronic patient records. Results After a 12 month period, there were significantly fewer outstanding jobs with no jobs waiting longer than 3 months and all jobs deemed appropriate for 'virutal' clinic follow up. There were no clinical risk incidents. Families have informally expressed their satisfaction with having direct hospital contact and promises made as an inpatient fulfilled. This implementation has become a permanent change wihtin the neonatal department.

Conclusion This quality improvement initiative shows that patients can be safely followed up 'virtually.' Communication and documentation between health professionals needs to be sound and of high quality. This can improve the quality of care delivered and improve satisfaction amongst our patients and families. 